\title{
Toward Development of Interleukin-11 as a Medical Countermeasure for Use in Radiological/Nuclear Emergencies
}

\author{
Martin Hauer-Jensen
}

Published online: 4 March 2014

(C) Springer Science+Business Media New York (Outside the USA) 2014

Since nuclear attacks and acts of radiological terrorism have emerged as serious civilian and military security risks over the past decade, medical countermeasures for use in radiological or nuclear emergencies are urgently needed.

In most representative radiation scenarios, the extent of injury to the highly radiosensitive rapidly proliferating stem and progenitor cells in bone marrow and the gastrointestinal (GI) tract epithelium dominate as determinants of survival. During the past decades, significant progress has been made in the management of bone marrow injury by the use of hematopoietic cytokines, blood transfusions, antimicrobial therapy, and stem cell reconstitution. In contrast, the management of GI radiation toxicity remains comparatively underdeveloped and largely symptomatic [1]. Therefore, because the GI system has gained in importance as a key determinant of survival, the need for medical countermeasures focused specifically on the GI tract has increased substantially.

GI acute radiation syndrome in humans occurs after total body irradiation (TBI) exposures at doses in excess of $6 \mathrm{~Gy}$. Destructive changes in the intestinal epithelium disrupt the mucosal barrier, with resultant severe secretory diarrhea, dehydration, and electrolyte imbalance. GI toxicity is also present after lower radiation doses, however, because translocation of bacteria from the intestinal lumen through a dysfunctional mucosal barrier occurs during a period when

M. Hauer-Jensen

Surgical Service, Central Arkansas Veterans Healthcare System, Little Rock, AR, USA

M. Hauer-Jensen ( $\square$ )

Division of Radiation Health, University of Arkansas for

Medical Sciences, 4301 West Markham, Slot 522-10,

Little Rock, AR 72205, USA

e-mail: mhjensen@life.uams.edu the immune system is severely compromised. In other words, sepsis from enteric bacteria ("gut-associated sepsis") is an important cause of lethality even after radiation exposure in the "hematopoietic" dose range (i.e., radiation exposures of less than 6 Gy in humans).

Development of medical countermeasures for use in radiological/nuclear emergencies is focused on pre-exposure and post-exposure interventions. Pre-exposure radioprophylactic or radioprotective countermeasures are interventions that enhance the resistance or tolerance of normal tissues to radiation or interfere directly with the initial radiochemical events. In contrast, post-exposure countermeasures interfere with downstream events, preventing or reducing the progression of radiation damage or facilitating the resolution of or recovery from radiation injury. Radioprotective or radioprophylactic countermeasures are important requirements for military personnel, first responders, and rescue and clean-up workers. In contrast, agents that are effective when administered hours to days after radiation exposure are needed following a civilian mass casualty event.

The threat of radiological/nuclear terrorism has brought to the foreground the need to develop effective, safe, and non-toxic agents to use as medical countermeasures in radiological and nuclear emergencies. In order to be useful as a medical countermeasure against radiation, a drug should ideally fulfill the criteria listed in Table 1. Moreover, if the indication as a medical countermeasure against radiation can be applied to a previously approved drug, the time to approval by the Food and Drug Administration (FDA) for the new indication can be significantly reduced since the formulation, safety, and manufacturing requirements have already been satisfied. Interleukin 11 (IL11), the focus of the article by Yang et al. [2] in this issue of Digestive Diseases and Sciences, is likely in this category. 
Table 1 Characteristics of the ideal radioprotector

Effective and safe (few side effects and drug interactions)

Stable and easy to produce (suitable for stockpiling)

Timing of administration not critical (wide therapeutic time window)

Useful in all operational environments (military operations, homeland defense, radiation accidents)

Favorable logistics (stable in the field, small weight/cube)

Useful (at least not harmful) in combined injury situations

No abuse potential

Interleukin 11 is a multifunctional cytokine of the interleukin 6 family with powerful anti-inflammatory properties, hematopoietic proliferative activity, and cytoprotective effects on intestinal crypt cells. Although recombinant human IL11 was developed and marketed to reduce chemotherapy-induced thrombocytopenia, it is of potential utility for the treatment of inflammatory bowel disease due to its anti-inflammatory and gut-protective properties. Systemic administration of IL11 improves the survival of crypt cells and reduces intestinal mucosal injury after exposure to TBI in mice [3, 4]. Nevertheless, although systemic administration of IL11 is well tolerated in animals, severe adverse effects in humans, including significant fluid retention, pleural effusion, and multisystem organ failure, have limited its clinical utility. Thus, although IL-11 is effective for both pre-exposure prophylaxis and post-exposure treatment $[3,5]$, its structure and route of administration must be modified in order to avoid excessive systemic toxicity.

Since exposure to neutron irradiation is very likely in a realistic nuclear event, the efficacy of potential medical countermeasures to neutron irradiation is of prime importance. Neutron irradiation has a higher relative biological effectiveness (RBE) than gamma- or X-rays. Therefore, a considerably lower neutron dose was used in the study by Yang et al. than in studies with gamma- or X-rays. The time-course of the survival curve confirms that the mice died from GI acute radiation syndrome, which usually causes death within 7 days of exposure. The effect of IL11 in vivo was relatively modest, restricted to a $\sim 0.5$ day increase of median survival time. Nonetheless, in this preliminary study, an entire range of neutron doses was not investigated, nor was the dose of IL11 optimized. Also, although suggestive histological examples are shown, more quantitative methods for assessing intestinal injury such as the crypt colony assay [6] or plasma citrulline concentrations [7] may have uncovered significant differences. On the other hand, apoptosis and necrosis in IEC-6 cells after neutron exposure, as measured with flow cytometry, was reduced by $\mathrm{Il}-11$ treatment.

That this initial study did not include dose optimization raises the question of how the dosage of IL11 $(500 \mu \mathrm{g} / \mathrm{kg})$ used was determined. It would have been very interesting, for example, to have measured systemic plasma IL11 concentrations compared to those in intestinal tissue. A 20-fold higher dose of IL11 given by oral gavage robustly increased whole mouse survival and intestinal crypt survival [5], suggesting that higher enterally delivered doses might have been more effective in the study by Yang et al.

Because medical countermeasures against radiation cannot be tested in humans, the FDA requires mechanistic information in addition to data supporting efficacy in two distinct animal species. The mechanistic data reported by Yang et al. relative to the MEK/ERK and PI3 K/Akt pathways are, therefore, of considerable interest and value. Although these pathways are involved in the endogenous regulation of IL11 expression [8], data suggesting that MEK inhibition and PI3 $\mathrm{K}$ inhibition antagonizes the beneficial effects of exogenous IL11 after neutron irradiation are novel and potentially important.

It is unlikely that a single therapy will be effective in mitigating both GI and hematologic acute radiation syndromes. Combined therapies will be necessary that are effective, non-toxic, and easily distributed. In this respect, IL11 as a countermeasure to protect the GI tract from lethal doses of neutron radiation has several attractive qualities, although it will be necessary to determine which modifications are necessary to avoid toxicity, provided IL11 is given systemically. If, on the other hand, oral administration of IL11 is considered, efficacy needs to be examined with neutron irradiation. Regardless of what route of administration is chosen, it would be prudent to test a range of neutron doses, to optimize the dose of IL11, and to determine whether the mechanistic data from the current study obtained in IEC-6 cells also applies in vivo.

Acknowledgments Dr. Hauer-Jensen receives support from the National Institutes of Health (U19 AI67798, R37 CA71382, R01 CA148679, R01 CA152667, and UL1 RR029884), the Biomedical Advanced Research and Development Authority (HHSO1002 01100045C), the Edward P. Evans Foundation, and the Veterans Administration.

\section{References}

1. Dainiak N, Gent RN, Carr Z, et al. Literature review and global consensus on management of acute radiation syndrome affecting nonhematopoietic organ systems. Disaster Med Public Health Prep. 2011;5:183-201.

2. Yang L, Wang R, Gao Y, et al. The protective role of interleukin11 against neutron radiation injury in mouse intestines via MEK/ ERK and PI3K/Akt dependent pathways. Dig Dis Sci. (Epub ahead of print). doi:10.1007/s10620-013-3015-0.

3. Potten CS. Interleukin-11 protects the clonogenic stem cells in murine small-intestinal crypts from impairment of their reproductive capacity by radiation. Int J Cancer. 1995;62:356-361.

4. Potten CS. Protection of the small intestinal clonogenic stem cells from radiation-induced damage by pretreatment with interleukin 
11 also increases murine survival time. Stem Cells. 1996;14:452-459.

5. Burnett AF, Biju PG, Lui H, Hauer-Jensen M. Oral interleukin 11 as a countermeasure to lethal total-body irradiation in a murine model. Radiat Res. 2013;180:595-602.

6. Withers HR, Elkind MM. Microcolony survival assay for cells of mouse intestinal mucosa exposed to radiation. Int J Radiat Biol Relat Stud Phys Chem Med. 1970;17:261-267.
7. Lutgens LCHW, Deutz NEP, Gueulette J, et al. Citrulline: a physiologic marker enabling quantitation and monitoring of epithelial radiation-induced small bowel damage. Int $J$ Radiat Oncol Biol Phys. 2003;57:1067-1074.

8. Shin SY, Choi C, Lee HG, Lim Y, Lee YH. Transcriptional regulation of the interleukin-11 gene by oncogenic Ras. Carcinogenesis. 2012;33:2467-2476. 